\title{
The urgency of severity and mortality risk assessments for COVID-19: A summary from death cases in Wuhan, China
}

\section{Feihong Yang}

Wuhan University Zhongnan Hospital

Jiaohong Gan

Wuhan University Zhongnan Hospital

Hao Zou

Wuhan University Zhongnan Hospital

Zhongxiang Zhang

Wuhan University Zhongnan Hospital

\section{Yan Zhao}

Wuhan University Zhongnan Hospital

Cheng Jiang

Wuhan University Zhongnan Hospital

Jian Xia ( $\sim$ jianjian_1998@sina.com )

Wuhan University Zhongnan Hospital

\section{Research article}

Keywords: COVID-19, ARDS, severity, mortality risk

Posted Date: April 16th, 2020

DOI: https://doi.org/10.21203/rs.3.rs-21792/v1

License: (9) (i) This work is licensed under a Creative Commons Attribution 4.0 International License. Read Full License 


\section{Abstract}

Background To investigate the clinical characteristics of 21 death cases and evaluate potential factors of disease severity and mortality risk in COVID-19.

Methods Retrospective analysis was used to study the clinical data of 21 death cases with COVID-19. The assessment of disease severity and mortality risk were conducted by APACHE II, SOFA, MuLBSTA and PSI scores.

Results The age was $66 \pm 14$ years-old and $15(71.4 \%)$ were men. $16(76.2 \%)$ patients had chronic medical illnesses. 12 (57.1\%) patients were overweight. Decreased lymphocytes were observed in $17(81.0 \%)$ patients on admission. Elevated D-dimer levels were noticed in $11(52.4 \%)$ patients and increased much more when pneumonia deteriorated. The initial APACHE II and SOFA scores demonstrated 18 (85.7\%) and $13(61.9 \%)$ patients in middle-risk levels, respectively. MuLBSTA and PSI scores after admission showed high-risk mortality in 13 (61.9\%) patients. Most patients developed sequent organ failure and finally caused death.

Conclusion Older, male, overweight patients, combined with chronic medical histories, continuous decreased lymphocyte proportion and increased D-dimer might have a higher risk of death. The combination of general scoring (SOFA) and pneumonia specific scoring (MuLBSTA and PSI) after admission might be more sensitive to assess the mortality risk for critical patients in COVID-19.

\section{Background}

Coronavirus disease 2019 (COVID-19) is an infectious disease which outbroke since early December, 2019. On Jan 20, 2020, it was identified that COVID-19 could spread from person to person through the respiratory tract by National Health Commission (NHC) of China. As of Mar 24, 2020, more than 370,000 patients suffered from this disease around the world. Severe acute respiratory syndrome coronavirus 2 (SARS-CoV-2), the pathogen of COVID-19, was found to cause acute respiratory distress syndrome (ARDS), myocardial injury, kidney and liver damages, coagulation abnormality [1, 2] and maybe central nervous system lesion (confirmed but unpublished by Beijing Ditan Hospital). All these organ dysfunctions accelerated disease progression and could lead to death[1, 3]. It has caused more than 18,000 deaths up to Mar 24 all over the world. The two homologous coronaviruses, severe acute respiratory syndrome coronavirus (SARS-CoV) and Middle East respiratory syndrome coronavirus (MERSCoV), have caused more than 1700 deaths in the past two decades, with mortality rates of more than $10 \%$ in SARS and 37\% in MERS[4]. SARS-CoV-2 seems to have a stronger infectivity and a relatively weaker lethality than SARS-CoV and MERS-CoV.

In a recent single-center study, SARS-CoV-2 caused a mortality rate up to $61.5 \%$ in critical patients[1]. Another single-center research pointed out the potential relationship between older age, respiratory failure, multiorgan dysfunction and mortality[5]. One multi-center retrospective cohort study analyzed risk factors for death in adults COVID-19, and identified that older age, elevated D-dimer levels and higher SOFA score 
on admission were associated with higher risk of in-hospital mortality[2]. These researches helped clinicians understand the clinical features of death cases and probable risk factors of mortality in COVID19 to some extent. However, under the pandemic situation and the relatively insufficient medical resources in the world, we need to find some useful methods or assessment tools to identify the severity and mortality risk in COVID-19 as early as possible.

In this retrospective, descriptive and single-center study, we enrolled 21 death cases who were confirmed COVID-19 and admitted to Zhongnan Hospital of Wuhan University. The clinical characteristics and several influential factors would be presented. The evaluations of disease severity and mortality risk would be calculated through different scoring systems, which could suggest a more cautious assessment and identification at early phase of disease. Thus, more intensive care and beneficial treatment will be offered to certain individuals at a high risk of progressing quickly to critical cases to reduce mortality and disability rate.

\section{Methods}

\section{Study design and participants}

In this retrospective and descriptive study, we enrolled 21 patients admitted to emergency intensive care unit (EICU) in Zhongnan Hospital of Wuhan University from Jan 3 to Feb 15, 2020. Zhongnan Hospital of Wuhan University is one of the major COVID-19 designated hospitals. All cases in the study were diagnosed as COVID-19 by laboratory detection according to World Health Organization interim guidance[6]. The study was approved by Zhongnan Hospital Ethics Committee (No.2020005) and written informed consent was waived due to the rapid emergence of this infectious disease. These patients have not been reported in any other submission.

\section{Procedures and Data collection}

We collected and scanned the electronic medical records of 21 death cases. Demographic, epidemiological, clinical, laboratory and radiological characteristics, nursing records, treatment and outcomes data were obtained and reorganized using a standardized data collection form (a modified form for severe acute respiratory infection clinical characterization shared by the International Severe Acute Respiratory and Emerging Infection Consortium). The key duration from the first symptoms to death were recorded. The assessment of disease severity and organ dysfunction were also brought into study. Acute Physiology and Chronic Health Evaluation II (APACHE II), sepsis-related organ failure assessment (SOFA), Multilobular infiltration, hypo-Lymphocytosis, Bacterial coinfection, Smoking history, hyper-Tension and Age (MuLBSTA) and pneumonia severity index (PSI) scores were calculated according to accepted standards since admission. The data (including images) were reviewed by two advanced physicians or radiologists to ensure accuracy. 


\section{Outcomes}

The primary outcomes were the early evaluations of disease severity and mortality risk through standard scoring systems. Various original and statistical data or images described in the above-mentioned procedures were also summarized and analyzed. All data were objective and certified.

\section{Statistical analysis}

Continuous variables were described as mean \pm standard deviation (SD) or median (25-75\% interquartile) and categorical variables as number (percentages). All statistical analyses were performed with SPSS software (version 25.0, IBM Inc).

\section{Results}

\section{Baseline Characteristics and Prehospital Treatment}

The study included 21 death cases with confirmed COVID-19, none of which had an exposure history to Huanan Seafood Market. As shown in Table 1, the mean age was $66 \pm 14$ years-old, and 10 (47.6\%) were older than 70 years-old. $15(71.4 \%)$ patients were men. 12 (57.1\%) patients were overweight by calculating BMI. Of the 21 patients, 16 (76.2\%) had coexisting chronic medical illnesses and 9 patients suffered more than one illness. Before admission, the most common symptoms were fever $(18,85.7 \%)$, shortness of breath $(16,76.2 \%)$ and dry cough $(11,52.4 \%)$. In patients with fever, $4(19.0 \%)$ had a highest temperature under $38.0^{\circ} \mathrm{C}, 6(28.6 \%)$ in $38.1-39.0^{\circ} \mathrm{C}$ and $4(19.0 \%)$ above $39.0^{\circ} \mathrm{C}$ before admission. The temperatures of another $4(19.0 \%)$ fever patients were vague and not included here. While, $14(66.7 \%)$ patients were in normothermia on admission. 19 (90.5\%) patients received treatment in outpatient department because of a lack of beds and $1(4.8 \%)$ patient accepted residential treatment in other hospital before admission. $13(61.9 \%)$ of these patients were treated with antibiotic drugs, $8(38.1 \%)$ with antiviral drugs, 1 (4.8\%) with systemic corticosteroid and 1 (4.8\%) with immunoglobulin. In the study, the durations from first symptoms to admission were $5 \pm 3$ days. 19 (90.5\%) patients were classified as severe status and $2(9.5 \%)$ as critical status on admission according to the guidelines for Diagnosis and Treatment of COVID-19 ( $7^{\text {th }}$ edition) published by NHC. Most patients showed tachypnea with a respiratory rate of $24 \pm 6$ times/minute and $2(9.5 \%)$ patients over 30 . We also analyzed the images within 24 hours before or immediately after admission to ascertain pulmonary lesions before the initiation of our treatment. All the 21 patients showed bilateral multiple ground-glass opacity on CT or X-ray scans. Bilateral injuries from superior to inferior lobes appeared in 19 (90.5\%) patients. The rest two patients showed abnormal regions either in superior or inferior fields. The involved area of 12 (57.1\%) patients exceeded $50 \%$, showing extensive effusion and consolidation.

\section{Vital Laboratory Parameters and Imaging Features}

On admission, leucocytes were below the normal range in $8(38.1 \%)$ patients and above the normal range in 3(14.3\%) patients (table 2). Procalcitonin (PCT), interleukin-6 (IL-6) and C-reactive protein (CRP) were 
generally higher than normal. Leucocytes and neutrophils increased in accordance with the infectious indicators when co-infection with bacterial outbroke. Lymphocytes below the normal range were observed in $17(81.0 \%)$ patients with a value of $0.7 \pm 0.5 \times 10^{\wedge} 9 / \mathrm{L}$ on admission. Also, platelets were below the normal range in 11 (52.4\%) patients. Lymphocyte percentage and platelets decreased gradually with inflammation progression (Figure 1), which was a feature of COVID-19 in the study. Decreased albumin levels were observed in most patients. Bio-markers reflecting organ functions are displayed on the Table 2. The maximum of high-sensitive troponin I (hs-Tnl) exceeded $50000 \mathrm{pg} / \mathrm{ml}$ in a patient with acute myocardial infarction. PT increased to a relative higher level as condition worsened (Figure 1). Elevated serum D-dimer were noticed in 11 (52.4\%) patients on admission and the proportion increased to $100 \%$ when pneumonia deteriorated.

Chest CT or X-ray was conducted at approximately 3-day intervals. Representative radiologic findings of two patients were presented in Figure 2. On admission, multiple ground-glass opacity and exudation appeared in bilateral subpleural areas. Lesions expanded rapidly just in several days and manifested as ground-glass opacity and air bronchograms coexisting with extensive consolidation or fibrous stripes. A white lung appearance could be always seen in end-stage COVID-19.

\section{Severity of Illness Scores}

In the study, we evaluated severity, progression and prognosis of patients' condition by figuring APACHE II, SOFA, MuLBSTA and PSI scores dynamically. In Table 3, we presented different levels of the four scores after admission, before ventilation and the maximum in the course. The initial APACHE II and SOFA scores were $14 \pm 3$ and 7 (5-8) respectively. 18 (85.7\%) and 13 (61.9\%) patients were in the middlerisk levels of each. Both APACHE II and SOFA scores increased before ventilation and more patients reached the high-risk levels in accordance with the deterioration of patients' condition and the urgent requirement for non-invasive or invasive ventilation. We found 13 patients were over 12 points of MuLBSTA on admission which predicted a high risk of death. But no significant fluctuation of MuLBSTA score was observed from admission to ventilation. Same changes were observed in PSI. The PSI score was 137 (102-146) after admission and 138 (125-157) before ventilation, with 13 (61.9\%) patients over 130 in the high-risk level in both stages. All the four scores reached a maximum just before death including the same 19 (90.5\%) patients in each high-risk level, with a median of APACHE II 26 (22-32) points, SOFA 14 (12-16) points, MuLBSTA 15 (13-16) points and PSI 163 (149-185) points, implying a very high mortality.

\section{Main Interventions and Complications}

Patients with COVID-19 were treated in isolation. In Table 4, all the patients received antiviral treatment after admission including intravenous potassium sodium dehydroandroan drographolide succinate and oral oseltamivir or lopinavir and ritonavir tablets, abidol hydrochloride tablets latter according to guidelines of NHC. Also, all patients were given empirical antibiotic treatment covering common pathogens and some atypical pathogens. In patients $(18,85.7 \%)$ with secondary bacterial infection or septic shock, higher-level and combined antibiotics such as carbapenems and anti-gram positive drugs 
were administered according to bacterial culture and drug sensitivity. $2(9.5 \%)$ patients were treated with antifungal drugs. 16 (76.2\%) patients received systemic corticosteroids with an initial dose of $1-2 \mathrm{mg} / \mathrm{kg}$ per day on the basis of patient's condition and $3(14.3 \%)$ patients were given large dose of immunoglobulins. Low molecular weight heparin was subcutaneously administered for prophylactic anticoagulation in patients of hypercoagulable state. 14 (67\%) patients received high-flow nasal cannula oxygen therapy. 8 (38.1\%) patients were give intermittently or continuous non-invasive ventilation for 1-11 days after a duration of 1 (0-5) day from admission. Invasive ventilation was required in 18 (85.7\%) patients after a duration of $4 \pm 3$ days from admission. A total of 19 patients were given norepinephrine to correct hypotension. 6 (28.6\%) patients received renal replacement therapy owing to renal failure, among whom 2 patients had a long-term regular hemodialysis for chronic renal diseases. Another 3 (14.3\%) patients had refractory hypoxaemia and received extracorporeal membrane oxygenation (ECMO) as salvage therapy, including one after successful cardiopulmonary resuscitation.

The total course from first symptoms to death was $18 \pm 8$ days with a duration of 12 (8-17) days in hospitalization. Patients died within $8 \pm 5$ days after invasive ventilation. In the disease progression, all patients were diagnosed as severe pneumonia followed by ARDS and a majority of patients $(19,90.5 \%)$ developed multiple organ dysfunction. Acute renal, liver and cardiac injury were developed in 17 (81.0\%), 12 (57.1\%) and 7 (33.3\%) patients respectively. Another 2 patients suffered fatal acute myocardial infarction or upper gastrointestinal and died in convalescence stage of pneumonia. All patients developed to coagulation abnormality. Secondary infections were observed and evolved into septic shock in $18(85.7 \%)$ patients. Hospital-acquired infection was noticed in 5 (23.8\%) patients. Multidrug-resistant cinetobacter baumannii infection was identified from sputum in $3(14.3 \%)$ patients, one of whom had a co-infection with escherichia coli. Corynebacterium striatum was identified in the blood culture of 1 (4.8\%) patient. Another patient was diagnosed as urinary tract infection with urine culture of candida glabrata.

\section{Discussion}

COVID-19 has spread in more than 100 countries up to late March, which is much more infectious and invasive than SARS and MERS[4]. The severe cases reached a peak of $11977(16.1 \%)$ on Feb 18th and gradually decreased later in China according to data published by NHC. The total mortality 4.0\% (March 26th) is much lower than SARS in 2003 and MERS in 2015[4]. However, the mortality in severe patients exceeded $60 \%$ in previous report[1]. In this single-center and retrospective study, we described and analyzed the demographic and clinical characteristics of 21 death cases with confirmed COVID-19 in EICU of Zhongnan Hospital of Wuhan University.

Males $(71.4 \%)$ were major victims here in this study similar with other researches in different hospitals[1, $2,5]$. The mean age was 66 years with almost a half over 70 years. Combining with COVID-19 studies in other centers during the same period, older males seem more susceptible to SARS-CoV-2 infection and 
are the primary members in severe group with poor prognosis[1,2, 5], which is supported by our data. Same findings were reported in previous SARS $[7,8]$ and MERS studies $[9,10]$. We found overweight adults account for more than half in the study, which was ignored before. We should also pay attention that a few patients suffered self-healing diarrhea during the course. The mean time from illness onset to hospital admission was 5 days, less than 11 days presented in the research conducted in Jinyintan Hospital and Wuhan Pulmonary Hospital[2]. 20 patients had received oral or intravenous medication treatments before admission including antibiotic and antiviral therapy mostly. However, no drugs seemed effective to delay the progression of COVID-19 in the early stage of the epidemic.

In laboratory tests, reduced lymphocytes, a characteristic index in SARS-CoV-2 infection, were observed in majority patients in our study, which is also mentioned in many other reports[3,11, 12]. We speculate the continuous lymphocyte proportion decrease may indicate excessive consumption by SARS-CoV-2 like the mechanism in SARS-CoV and MERS-CoV infection[13, 14]. Lymphopenia seemed more common in severe patients[2, 3]and might reflect the severity and higher mortality of COVID-19. Lymphocyte subpopulation was detected and both CD4-positive and CD8-positive T lymphocytes decreased significantly. Damage of T lymphocytes and inhibition of the cellular immune system may contribute great to the deterioration. Levels of serum IL-6 increased rapidly in a short period, which could be explained by the active inflammatory storm $[5,6]$. In this study, we detected reduced platelets on admission and progressive decline during hospitalization, which were previously demonstrated to have a relation with severe inflammation[15, 16]. Urea and creatinine rose in different degrees represented renal dysfunction because of virus invasion, hypoxaemia, hypoperfusion or original kidney diseases. Coagulation dysfunction was observed in almost all cases marked by prolonged PTs and significant increased serum D-dimer concentrations.

Despite of many clinical assessment and prediction scores, there is no standard recommendation to predict the severity and risk of mortality for patient with viral pneumonia up to now. Viral pneumonia, including COVID-19, might progress rapidly to critical illness and develop to ARDS, multiple organ failure and even death. APACHE II scoring system has been widely used to assess patient severity and predict outcome in critical ill patients[17]. SOFA scoring has also been used to predict prognosis and assist the diagnosis of sepsis/septic shock in intensive care unit (ICU). In our study, the APACHE II and SOFA scores on admission were $14 \pm 3$ and 7 (5-8) respectively. The highest APACHE II and SOFA scores were 26 (2232 ) and 14 (12-16), respectively. They both increased along with the progression of disease. The SOFA score was similar with the non-survivors at ICU admission in one study about COVID-19 critically ill patients[3, 6], however, the APACHE II score was similar with the survivors. Another study found that higher SOFA score at admission was associated with higher odds of death in COVID-19 patients[2]. This study did not present the APACHE II score in article. SOFA scores, including mean and highest SOFA scores, are both good predictors for prognosis. Independent of the initial score, an increase of SOFA score during the first $48 \mathrm{~h}$ predicts a mortality rate of $50 \%$ [18]. On the other hand, SOFA score is the diagnostic marker for sepsis/septic shock [19]. In our study, 18 (85.7\%) of patients developed septic shock. As the study demonstrated that more than $50 \%$ of COVID-19 patients developed sepsis[2], which reminded us the possibility of virus induced sepsis syndrome besides SARS-CoV-2 infection combined bacterial 
infection. APACHE II score $10-20$ suggests the mortality rate of about $50 \%$, and above 20 suggests the mortality rate of about $80 \%$. Although existing difference between studies aforementioned, we still considered that APACHE II score had its general predictability for outcome in COVID-19, which might be less sensitive enough than SOFA score.

In our study, we conducted two special scores to predict the probability of mortality in pneumonia, MuLBSTA[20] and PSI [21]. Both score results in our study showed that 13 (61.9\%) patients had the high risk of mortality at admission. The highest scores increased to 15 (13-16) (MuLBSTA) and 163 (149-185) (PSI), respectively, accounting for 19 (90.5\%) patients. Up to now, there is few research analyzing pneumonia special severity scoring system in SARS-CoV-2 infected pneumonia. One research about COVID-19 mortality analysis found that CURB-65 score was significantly higher in non-survival groups than survival groups[2]. Our study identified the usefulness of MuLBSTA and PSI scoring systems in predicting the risk of mortality in COVID-19. However, the accurate sensitivity and specificity of those pneumonia special severity scoring systems needs further research and verification.

All the death cases showed complications, mainly including ARDS (100\%), septic shock (85.7\%), acute kidney injury (81.0\%), liver dysfunction (57.1\%) and acute cardiac injury (33.3\%), which had been also observed in similar researches $[2,5,6]$. The primary cause of progressive impact on multiorgan by SARSCoV-2 is that the binding receptor for SARS-CoV-2, ACE2 mainly exists in blood vessels and lung alveolar type II (AT2) epithelial cells [22]. And ACE2 also exists in the heart, kidney, liver and so on. Thus, the viral infection may stimulate the immune cells to release pro-inflammatory cytokines and damage the target organs, even causing death.

The treatments for COVID-19 mainly covered anti-pathogen therapy, different kinds of oxygen therapy, glucocorticoid therapy, immunoglobulin therapy, and advanced life support for organ function, which were similar with published researches[2, 5, 6, 11]. 9 (42.9\%) patients received low molecular heparin therapy in our study. During the treatment of the COVID-19 patients, coagulation abnormalities can be observed through laboratory findings mostly, including D-dimer, prothrombin time and so on, but not all the cases may develop to some detectable relative clinical manifestations. In a large retrospective cohort study about COVID-19, researchers found that elevated D-dimer, greater than $1 \mu \mathrm{g} / \mathrm{L}$, at admission was a risk factor for mortality in adult patients with COVID-19 [2]. The final impacts of coagulation abnormalities could be understood according to the lung biopsy conducted by Shenzhen Third People's Hospital [23]. The whole lung tissue displayed a diffuse congestive appearance with variable degrees of hemorrhagic pulmonary infarction and microthrombosis formation prominently presented in outer edge of the lung. The biopsy also pointed out that hemorrhagic necrosis in the outer edge of the lung might be the origination of COVID-19 and one of the main causes of death in severe patients. Coagulation abnormalities can be observed in severe pneumonia and sepsis. In a study of community-acquired pneumonia, D-dimer had been found to elevate persistently in $86.5 \%$ of patients, even among the least ill cases[24]. The research about patients with infection or sepsis identified in the emergency department showed that high levels of D-dimer was associated with 28-day mortality[25]. These findings highlighted 
the complexity of the coagulation response to viral infection and corresponding coagulation-based therapeutics.

\section{Limitations}

There are several limitations in this study. Firstly, this is a retrospective, descriptive, single-center study with a small sample. The further large cohort study or some random controlled trials are needed to confirm our findings. Secondly, some cases had incomplete or missing data, including outpatient information, laboratory results in certain periods, sequential images and so on, which would influence the analysis accuracy in the study.

\section{Conclusions}

Older, male, overweight patients, combined with chronic medical histories, continuous decreased lymphocyte proportion, and increased serum D-dimer levels might have a higher risk of death. The combination of general scoring (SOFA score) and pneumonia specific scoring (MuLBSTA score and PSI score) after admission might be more sensitive to assess the risk of mortality for critically ill patients in COVID-19. We suggested a high attention and further study to coagulation abnormalities and relevant definitive therapy in COVID-19.

\section{Declarations}

Ethics approval and consent to participate The study was approved by Zhongnan Hospital Ethics Committee (No.2020005) and written informed consent was waived due to the rapid emergence of this infectious disease.

Consent to publish I am authorized by my co-authors to enter into these arrangements.

I warrant, on behalf of myself and my co-authors, that:1. the article is original, has not been formally published in any other peer-reviewed journal, is not under consideration by any other journal and does not infringe any existing copyright or any other third party rights; 2 . We are the sole author(s) of the article and have full authority to enter into this agreement and in granting rights to BMC are not in breach of any other obligation;3. the article contains nothing that is unlawful, libellous, or which would, if published, constitute a breach of contract or of confidence or of commitment given to secrecy;4. We have taken due care to ensure the integrity of the article. To my/our - and currently accepted scientific - knowledge all statements contained in it purporting to be facts are true and any formula or instruction contained in the article will not, if followed accurately, cause any injury, illness or damage to the user.

I, and all co-authors, agree that the article, if editorially accepted for publication, shall be licensed under the Creative Commons Attribution License 4.0. In line with BMC's Open Data Policy, data included in the article shall be made available under the Creative Commons 1.0 Public Domain Dedication waiver, unless otherwise stated. If the law requires that the article be published in the public domain, I/we will notify 
BMC at the time of submission, and in such cases not only the data but also the article shall be released under the Creative Commons 1.0 Public Domain Dedication waiver. For the avoidance of doubt it is stated that sections 1 and 2 of this license agreement shall apply and prevail regardless of whether the article is published under Creative Commons Attribution License 4.0 or the Creative Commons 1.0 Public Domain Dedication waiver.

\section{Availability of data and materials Not applicable}

Competing interests All authors declare no competing interests.

Funding This study was supported by the Emergency Diaggnostic \& Therapeutic Center of Central China, Hubei Clinical Research Center for Emergency and Resuscitation and Special Science and Technology Project of Hubei province, China (No.2020FCA023).

Authors' Contributions Jian Xia, Cheng Jiang and Yan Zhao had the idea for and designed the study and had full access to all data in the study and take responsibility for the integrity of the data and the accuracy of the data analysis. Feihong Yang and Jiaohong Gan contributed to writing of the report. Cheng Jiang contributed to critical revision of the report. Hao Zou and Zhongxiang Zhang contributed to the statistical analysis. All authors contributed to data acquisition, data analysis, or data interpretation, and reviewed and approved the final version.

Acknowledgements This work is funded by the Emergency Diaggnostic \& Therapeutic Center of Central China, Hubei Clinical Research Center for Emergency and Resuscitation and Special Science and Technology Project of Hubei province, China (No.2020FCA023).

We acknowledge all the health-care workers involved in the diagnosis and treatment of these patients.

\section{References}

1. Yang X, Yu Y, Xu J, Shu H, Xia Ja, Liu H, Wu Y, Zhang L, Yu Z, Fang M et al: Clinical course and outcomes of critically ill patients with SARS-CoV-2 pneumonia in Wuhan, China: a singlecentered, retrospective, observational study. The Lancet Respiratory Medicine 2020.

2. Zhou F, Yu T, Du R, Fan G, Liu Y, Liu Z, Xiang J, Wang Y, Song B, Gu X et al: Clinical course and risk factors for mortality of adult inpatients with COVID-19 in Wuhan, China: a retrospective cohort study. The Lancet 2020.

3. Chen N, Zhou M, Dong X, Qu J, Gong F, Han Y, Qiu Y, Wang J, Liu Y, Wei Y et al: Epidemiological and clinical characteristics of 99 cases of 2019 novel coronavirus pneumonia in Wuhan, China: a descriptive study. The Lancet 2020, 395(10223):507-513.

4. Song Z, Xu Y, Bao L, Zhang L, Yu P, Qu Y, Zhu H, Zhao W, Han Y, Qin C: From SARS to MERS, Thrusting Coronaviruses into the Spotlight. Viruses 2019, 11(1). 
5. Zhang B, Zhou X, Qiu Y, Feng F, Feng J, Jia Y, Zhu H, Hu K, Liu J, Liu Z et al: Clinical characteristics of 82 death cases with COVID-19. medRxiv 2020:2020.2002.2026.20028191.

6. Huang C, Wang Y, Li X, Ren L, Zhao J, Hu Y, Zhang L, Fan G, Xu J, Gu X et al: Clinical features of patients infected with 2019 novel coronavirus in Wuhan, China. The Lancet 2020, 395(10223):497506.

7. Channappanavar R, Fett C, Mack M, Ten Eyck PP, Meyerholz DK, Perlman S: Sex-Based Differences in Susceptibility to Severe Acute Respiratory Syndrome Coronavirus Infection. J Immuno/ 2017, 198(10):4046-4053.

8. Li CS, Pan SF: [Analysis and causation discussion of 185 severe acute respiratory syndrome dead cases]. Zhongguo Wei Zhong Bing Ji Jiu Yi Xue 2003, 15(10):582-584.

9. Badawi A, Ryoo SG: Prevalence of comorbidities in the Middle East respiratory syndrome coronavirus (MERS-CoV): a systematic review and meta-analysis. Int $J$ Infect Dis 2016, 49:129-133.

10. Chen X, Chughtai AA, Dyda A, Maclntyre CR: Comparative epidemiology of Middle East respiratory syndrome coronavirus (MERS-CoV) in Saudi Arabia and South Korea. Emerg Microbes Infect 2017, 6(6):e51.

11. Guan WJ, Ni ZY, Hu Y, Liang WH, Ou CQ, He JX, Liu L, Shan H, Lei CL, Hui DSC et al: Clinical Characteristics of Coronavirus Disease 2019 in China. N Engl J Med 2020.

12. Wang D, Hu B, Hu C, Zhu F, Liu X, Zhang J, Wang B, Xiang H, Cheng Z, Xiong Y et al: Clinical Characteristics of 138 Hospitalized Patients With 2019 Novel Coronavirus-Infected Pneumonia in Wuhan, China. JAMA 2020.

13. Gu J, Gong E, Zhang B, Zheng J, Gao Z, Zhong Y, Zou W, Zhan J, Wang S, Xie Z et al: Multiple organ infection and the pathogenesis of SARS. J Exp Med 2005, 202(3):415-424.

14. Liu WJ, Zhao M, Liu K, Xu K, Wong G, Tan W, Gao GF: T-cell immunity of SARS-CoV: Implications for vaccine development against MERS-CoV. Antiviral Res 2017, 137:82-92.

15. Outinen TK, Laine OK, Makela S, Porsti I, Huhtala H, Vaheri A, Mustonen J: Thrombocytopenia associates with the severity of inflammation and variables reflecting capillary leakage in Puumala Hantavirus infection, an analysis of 546 Finnish patients. Infect Dis (Lond) 2016, 48(9):682-687.

16. Claushuis TA, van Vught LA, Scicluna BP, Wiewel MA, Klein Klouwenberg PM, Hoogendijk AJ, Ong DS, Cremer OL, Horn J, Franitza M et al: Thrombocytopenia is associated with a dysregulated host response in critically ill sepsis patients. Blood 2016, 127(24):3062-3072.

17. Knaus WA, Draper EA, Wagner DP, Zimmerman JE: APACHE Il: a severity of disease classification system. Crit Care Med 1985, 13(10):818-829.

18. Ferreira FL, Bota DP, Bross A, Mélot C, Vincent J-L: Serial Evaluation of the SOFA Score to Predict Outcome in Critically III Patients. JAMA 2001, 286(14):1754-1758.

19. Singer M, Deutschman CS, Seymour CW, Shankar-Hari M, Annane D, Bauer M, Bellomo R, Bernard GR, Chiche J-D, Coopersmith CM et al: The Third International Consensus Definitions for Sepsis and Septic Shock (Sepsis-3). JAMA 2016, 315(8):801-810. 
20. Guo L, Wei D, Zhang X, Wu Y, Li Q, Zhou M, Qu J: Clinical Features Predicting Mortality Risk in Patients With Viral Pneumonia: The MuLBSTA Score. Front Microbio/ 2019, 10:2752-2752.

21. Fine MJ, Auble TE, Yealy DM, Hanusa BH, Weissfeld LA, Singer DE, Coley CM, Marrie TJ, Kapoor WN: A Prediction Rule to Identify Low-Risk Patients with Community-Acquired Pneumonia. New England Journal of Medicine 1997, 336(4):243-250.

22. Hoffmann $M$, Kleine-Weber $H$, Krüger $N$, Müller M, Drosten $C$, Pöhlmann $S$ : The novel coronavirus 2019 (2019-nCoV) uses the SARS-coronavirus receptor ACE2 and the cellular protease TMPRSS2 for entry into target cells. bioRxiv 2020:2020.2001.2031.929042.

23. Luo WY, H.; Gou, J.; Li, X.; Sun, Y.; Li, J.; Liu, L. : Clinical Pathology of Critical Patient with Novel Coronavirus Pneumonia (COVID-19). Preprints 2020.

24. Milbrandt EB, Reade MC, Lee M, Shook SL, Angus DC, Kong L, Carter M, Yealy DM, Kellum JA, Gen IMSI: Prevalence and significance of coagulation abnormalities in community-acquired pneumonia. Mol Med 2009, 15(11-12):438-445.

25. Rodelo JR, De la Rosa G Fau - Valencia ML, Valencia MI Fau - Ospina S, Ospina S Fau - Arango CM, Arango Cm Fau - Gomez Cl, Gomez Ci Fau - Garcia A, Garcia A Fau - Nunez E, Nunez E Fau - Jaimes FA, Jaimes FA: D-dimer is a significant prognostic factor in patients with suspected infection and sepsis. (1532-8171 (Electronic)).

\section{Tables}




\begin{tabular}{|c|c|}
\hline Clinical characteristics & Patients $(n=21)$ \\
\hline Age, years & $66 \pm 14$ \\
\hline \multicolumn{2}{|l|}{ Sex } \\
\hline Male & $15(71.4 \%)$ \\
\hline Female & $6(28.6 \%)$ \\
\hline \multicolumn{2}{|l|}{ History of smoking } \\
\hline Positive & $3(14.3 \%)$ \\
\hline Negative & $18(85.7 \%)$ \\
\hline Body mass index, $\mathrm{kg} / \mathrm{m}^{2}$ & $25.1 \pm 4.5$ \\
\hline$<18.5$ & $2(9.5 \%)$ \\
\hline $18.5-23.9$ & $7(33.3 \%)$ \\
\hline$>23.9$ & $12(57.1 \%)$ \\
\hline \multicolumn{2}{|l|}{ Chronic medical illness } \\
\hline Cardiovascular diseases & $9(42.9 \%)$ \\
\hline Urinary system diseases & $5(23.8 \%)$ \\
\hline Cerebral vascular diseases & $4(19.0 \%)$ \\
\hline Lung diseases & $4(19.0 \%)$ \\
\hline Diabetes mellitus & $3(14.3 \%)$ \\
\hline \multicolumn{2}{|l|}{ Major symptoms on admission } \\
\hline Fever & $18(85.7 \%)$ \\
\hline Shortness of breath & $16(76.2 \%)$ \\
\hline Dry cough & $11(52.4 \%)$ \\
\hline More than one sign and symptom & $19(90.5 \%)$ \\
\hline Fever, dry cough and shortness of breath & $7(33.3 \%)$ \\
\hline Highest temperature before admission & $38.6 \pm 0.6$ \\
\hline$<37.3^{\circ} \mathrm{C}$ & $3(14.3 \%)$ \\
\hline $37.3-38^{\circ} \mathrm{C}$ & $4(19.0 \%)$ \\
\hline $38.1-39^{\circ} \mathrm{C}$ & $6(28.6 \%)$ \\
\hline$>39^{\circ} \mathrm{C}$ & $4(19.0 \%)$ \\
\hline Missing & $4(19.0 \%)$ \\
\hline \multicolumn{2}{|l|}{ Fever on admission } \\
\hline Yes & $7(66.7 \%)$ \\
\hline No & $14(33.3 \%)$ \\
\hline \multicolumn{2}{|l|}{ Treatment before admission } \\
\hline Outpatient service & $19(90.5 \%)$ \\
\hline Hospitalization elsewhere & $1(4.8 \%)$ \\
\hline Antibiotic therapy & $13(61.9 \%)$ \\
\hline Antiviral therapy & $8(38.1 \%)$ \\
\hline Systemic corticosteroid therapy & $1(4.8 \%)$ \\
\hline Immunoglobulin therapy & $1(4.8 \%)$ \\
\hline No treatment & $1(4.8 \%)$ \\
\hline Days from first symptoms to admission & $5 \pm 3$ \\
\hline Days from first symptoms to death & $18 \pm 8$ \\
\hline Days from admission to death (hospital stay) & $12(8-17)$ \\
\hline Days from admission to non-invasive ventilation & $1(0-5)$ \\
\hline Days from admission to invasive ventilation & $4 \pm 3$ \\
\hline Days from invasive ventilation to death & $8 \pm 5$ \\
\hline \multicolumn{2}{|l|}{ Disease severity status } \\
\hline Severe & 19 (90.5\%) \\
\hline Critical & $2(9.5 \%)$ \\
\hline Respiratory rate on admission (times/min) & $24 \pm 6$ \\
\hline
\end{tabular}

Page 13/17 


\begin{tabular}{|c|c|}
\hline$>30$ times $/ \mathrm{min}$ & $2(9.5 \%)$ \\
\hline \multicolumn{2}{|l|}{ Imaging features on admission } \\
\hline Bilateral involvement & $21(100.0 \%)$ \\
\hline All fields & 19 (90.5\%) \\
\hline Upper fields & $1(4.8 \%)$ \\
\hline Lower fields & $1(4.8 \%)$ \\
\hline Involved area > 50\% & $12(57.1 \%)$ \\
\hline Involved area $<50 \%$ & $9(42.9 \%)$ \\
\hline
\end{tabular}

Table 1. Baseline clinical characteristics on admission of 21 patients with confirmed COVID-19.

\begin{tabular}{|c|c|c|c|c|}
\hline ndings & Admission & Increase $/$ decrease $(n=$ & Maximum & Minimum \\
\hline $\mathrm{L}, 3.5-9.5)$ & $10.5 \pm 8.2$ & $8(38.1 \%) / 3(14.3 \%)$ & $20.8 \pm 8.4$ & $7.7 \pm 5.1$ \\
\hline$\left(\times 10^{\wedge} 9 / \mathrm{L}, 1.1-3.2\right)$ & $0.7 \pm 0.5$ & $0 / 17(81.0 \%)$ & $1.3 \pm 0.9$ & $0.3 \pm 0.3$ \\
\hline sroportion $(\%, 20.0-40.0)$ & $9.9 \pm 7.8$ & $0 / 19(90.5 \%)$ & $11.7 \pm 8.1$ & $2.3 \pm 1.6$ \\
\hline$\left.<10^{\wedge} 9 / \mathrm{L}, 1.8-6.3\right)$ & $9.2 \pm 7.9$ & $10(47.6 \%) / 0$ & $19.3 \pm 8.3$ & $6.6 \pm 4.6$ \\
\hline $\mathrm{g} / \mathrm{L}, 115-150)$ & $129.1 \pm 24.4$ & $3(14.3 \%) / 4(19.0 \%)$ & $134.4 \pm 24.5$ & $108.0 \pm 26.1$ \\
\hline$\left.\wedge^{\wedge} 9 / \mathrm{L}, 125-350\right)$ & $140 \pm 66.2$ & $0 / 11(52.4 \%)$ & $173.2 \pm 74.1$ & $70.9 \pm 54.1$ \\
\hline $0-0.05)$ & $3.1 \pm 5.6$ & $21(100.0 \%) / 0$ & $11.3 \pm 14.8$ & $1.1 \pm 1.9$ \\
\hline$)-7)$ & $77.0 \pm 23.7$ & $5 / 5(100 \%) / 0$ & $472.5 \pm 405.8$ & $33.3 \pm 20.5$ \\
\hline-10$)$ & $125.4 \pm 74.0$ & $20(95.2 \%) / 0$ & $230.9 \pm 107.0$ & $88.9 \pm 85.6$ \\
\hline 5) & $38.8 \pm 20.1$ & $7(33.3 \%) / 0$ & $125.1 \pm 237.7$ & $22.6 \pm 10.8$ \\
\hline 35) & $66.0 \pm 33.9$ & $16(76.2 \%) / 0$ & $309.6 \pm 733.5$ & $33.6 \pm 18.1$ \\
\hline $\mathrm{I}(\mu \mathrm{mol} / \mathrm{L}, 5-21)$ & $16.6 \pm 6.7$ & $6(28.6 \%) / 0$ & $30.1 \pm 16.4$ & $13.6 \pm 6.1$ \\
\hline in $(\mu \mathrm{mol} / \mathrm{L}, 0-7)$ & $8.4 \pm 5.0$ & $9(42.9 \%) / 0$ & $16.4 \pm 11.4$ & $4.9 \pm 3.1$ \\
\hline$(\mathrm{g} / \mathrm{L}, 65-85)$ & $61.2 \pm 8.1$ & $0 / 14(66.7 \%)$ & $65.8 \pm 7.8$ & $53.6 \pm 7.1$ \\
\hline $40-55)$ & $32.1 \pm 5.6$ & $0 / 20(95.2 \%)$ & $34.9 \pm 3.4$ & $25.2 \pm 4.6$ \\
\hline l/L, 3.9-6.1) & $9.9 \pm 6.1$ & $15(71.4 \%) / 1(4.8 \%)$ & $15.7 \pm 7.1$ & $7.9 \pm 2.9$ \\
\hline , 2.8-7.6) & $10.1 \pm 7.2$ & $10(47.6 \%) / 0$ & $22.6 \pm 11.8$ & $8.7 \pm 6.8$ \\
\hline $\mathrm{mol} / \mathrm{L}, 49-90)$ & $168.5 \pm 251.1$ & $9(42.9 \%) / 0$ & $333.9 \pm 350.3$ & $113.6 \pm 157.9$ \\
\hline $3.5-5.3)$ & $4.0 \pm 0.7$ & $2(9.5 \%) / 6(28.6 \%)$ & $5.1 \pm 0.9$ & $3.7 \pm 0.7$ \\
\hline 137-147) & $137.8 \pm 9.5$ & $3(14.3 \%) / 10(47.6 \%)$ & $152.3 \pm 13.5$ & $135.6 \pm 7.9$ \\
\hline $0-26.2)$ & $504.6 \pm 1623.63$ & $7(33.3 \%) / 0$ & $1283.4 \pm 2110.4$ & $86.7 \pm 221.8$ \\
\hline L, 0-6.6) & $3.3 \pm 3.2$ & $2(9.5 \%) / 0$ & $15.8 \pm 22.1$ & $1.8 \pm 2.0$ \\
\hline $0-100)$ & $166.6 \pm 188.2$ & $6(28.6 \%) / 0$ & $504.8 \pm 567.4$ & $157.5 \pm 162.1$ \\
\hline j) & $13.6 \pm 2.3$ & $11(52.4 \%) / 0$ & $20.2 \pm 12.4$ & $12.4 \pm 1.5$ \\
\hline $\mathrm{lg} / \mathrm{dL}, 238-498)$ & $452.7 \pm 116$ & $5(23.8 \%) / 0$ & $546.0 \pm 99.4$ & $314.6 \pm 140.0$ \\
\hline $\mathrm{nL}, 0-500)$ & $2726.4 \pm 4538.2$ & $11(52.4 \%) / 0$ & $\begin{array}{l}24311.9 \\
\pm 36589.9 \\
\end{array}$ & $\begin{array}{l}1654.2 \\
\pm 3162.1 \\
\end{array}$ \\
\hline
\end{tabular}

Table 2. Laboratory results of 21 patients including the baseline values on admission and the maximum, minimum values during hospitalization. 


\begin{tabular}{llll}
\hline Scores & \multicolumn{1}{c}{ Admission } & Before ventilation & Maximum \\
\hline APACHE II & $14 \pm 3$ & $17 \pm 4$ & $26(22-32)$ \\
$<10$ & $1(4.8 \%)$ & 0 & 0 \\
\hline $10-19$ & $18(85.7 \%)$ & $13(61.9 \%)$ & $2(9.5 \%)$ \\
\hline$\geq 20$ & $2(9.5 \%)$ & $8(38.1 \%)$ & $19(90.5 \%)$ \\
\hline SOFA & $7(5-8)$ & $8(7-12)$ & $14(12-16)$ \\
\hline $0-5$ & $8(38.1 \%)$ & $2(9.5 \%)$ & 0 \\
\hline $6-10$ & $13(61.9 \%)$ & $11(52.4 \%)$ & $2(9.5 \%)$ \\
\hline$>11$ & 0 & $8(38.1 \%)$ & $19(90.5 \%)$ \\
\hline MuLBSTA & $13 \pm 2$ & $13 \pm 2$ & $15(13-16)$ \\
\hline$>12$ (High risk) & $13(61.9 \%)$ & $14(66.7 \%)$ & $19(90.5)$ \\
\hline$<12$ (Low risk) & $8(38.1 \%)$ & $7(33.3 \%)$ & $2(9.5 \%)$ \\
\hline PSI & $137(102-146)$ & $138(125-157)$ & $163(149-185)$ \\
\hline $71-90$ & $3(14.3 \%)$ & $1(4.8 \%)$ & 0 \\
\hline $91-130$ & $5(23.8 \%)$ & $7(33.3 \%)$ & $2(9.5 \%)$ \\
\hline$>130$ & $13(61.9 \%)$ & $13(61.9 \%)$ & $19(90.5 \%)$ \\
\hline
\end{tabular}

Table 3. Severity assessment of APACHE II, SOFA, MuLBSTA and PSI scores on admission, before ventilation and the maximum value during hospitalization in 21 patients.

\begin{tabular}{ll}
\hline Main intervention and complications & Patients (n=21) \\
\hline Main interventions & $21(100 \%)$ \\
Antiviral therapy & $21(100 \%)$ \\
Antibiotic therapy & $1(4.8 \%)$ \\
Antifungal therapy & $16(76.2 \%)$ \\
Systemic corticosteroids therapy & $3(14.3 \%)$ \\
Immunoglobulin therapy & $9(42.9 \%)$ \\
Low molecular heparin therapy & $14(66.7 \%)$ \\
High-flow nasal cannula oxygen therapy & \\
Mechanical ventilation & $8(38.1 \%)$ \\
Non-invasive mechanical ventilation & $18(85.7 \%)$ \\
Invasive mechanical ventilation & $6(28.6 \%)$ \\
Continuous renal replacement therapy & $3(14.3 \%)$ \\
Extracorporeal membrane oxygenation & $19(90.0 \%)$ \\
Norepinephrine therapy & \\
Main complications & $21(100 \%)$ \\
Acute respiratory distress syndrome & $19(90.5 \%)$ \\
Multiple organ dysfunction syndrome & $17(81.0 \%)$ \\
Acute kidney injury & $12(57.1 \%)$ \\
Liver dysfunction & $7(33.3 \%)$ \\
Acute cardiac injury & $18(85.7 \%)$ \\
Coagulation abnormality & $5(23.8 \%)$ \\
\hline Septic shock & \\
Hospital-acquired infection & \\
\hline & \\
\hline
\end{tabular}

Table 4. Main Interventions and complications of 21 patients during hospitalization 


\section{Figures}
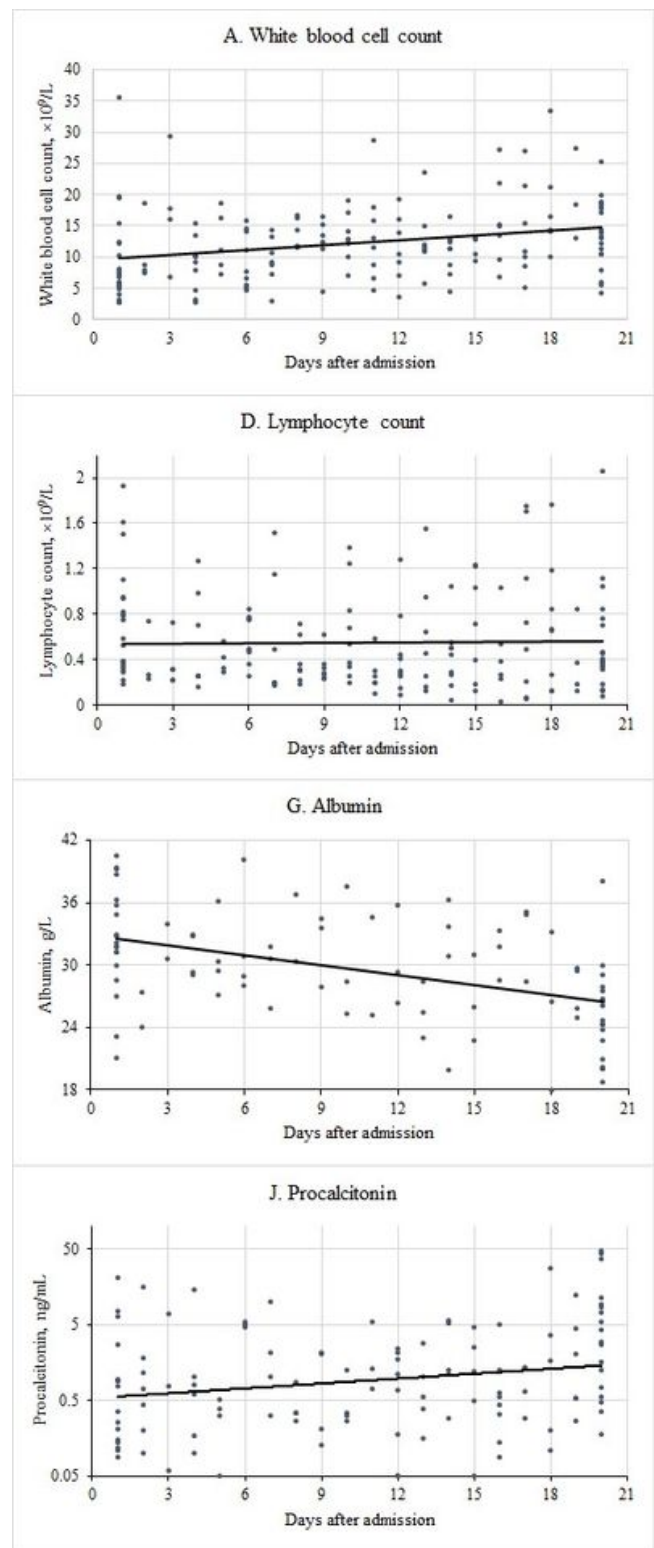
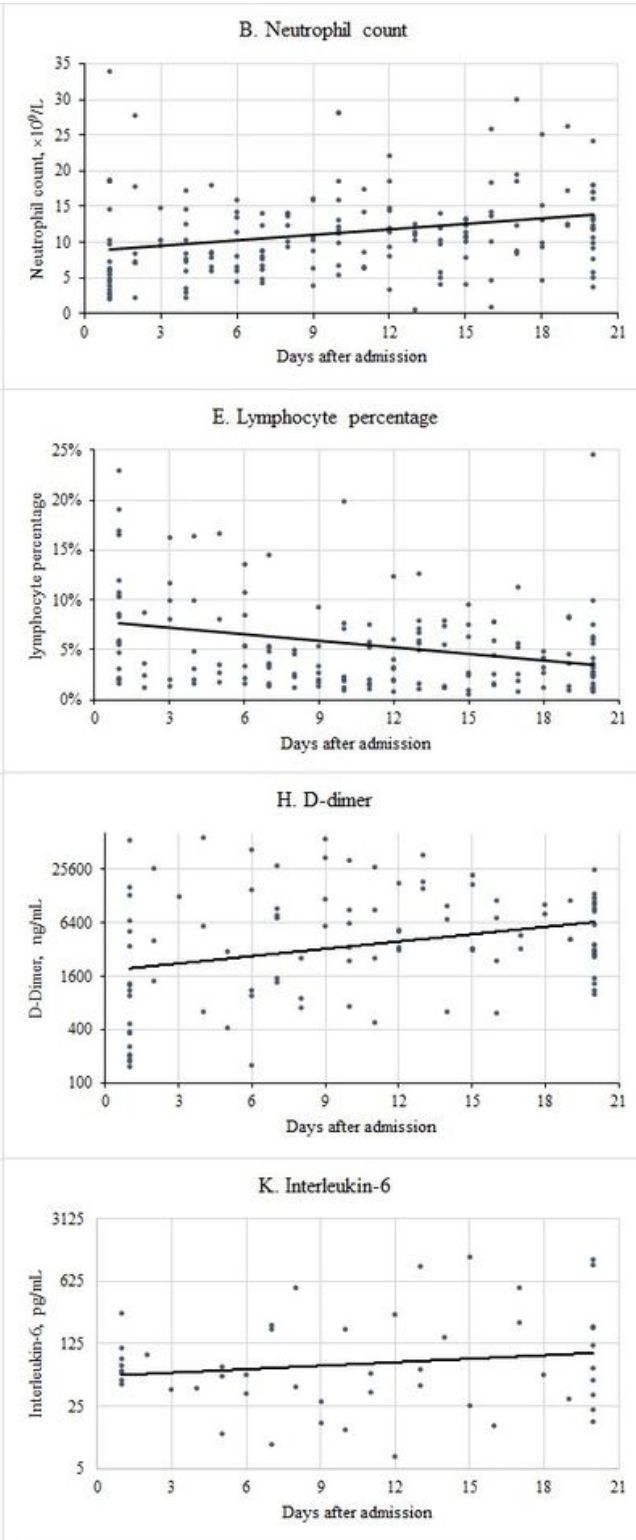
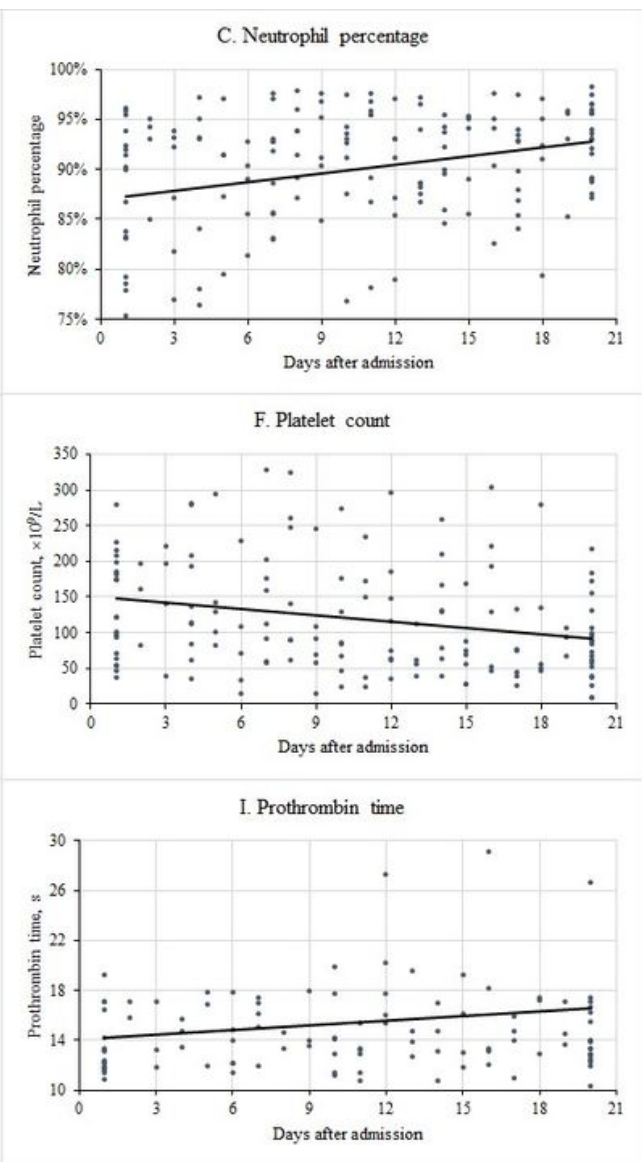

\section{Figure 1}

Results of laboratory examination during hospitalization 


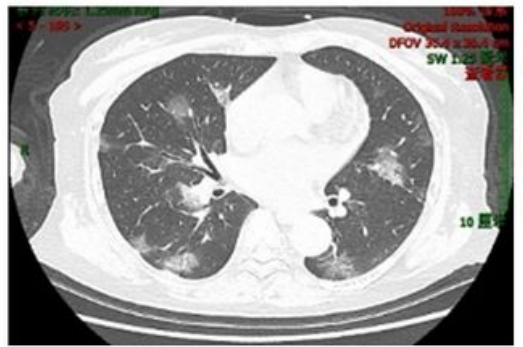

A

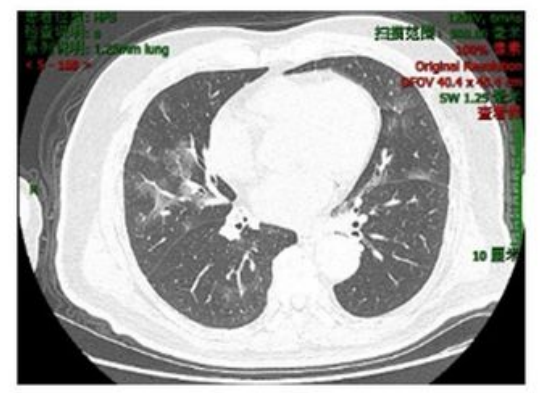

D

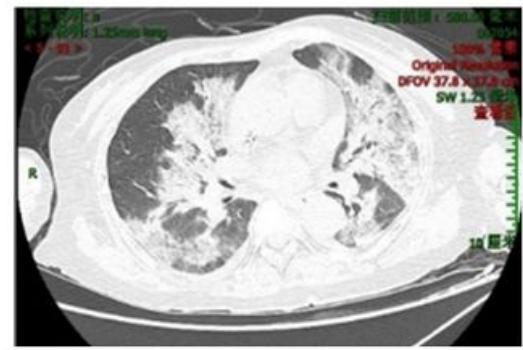

B

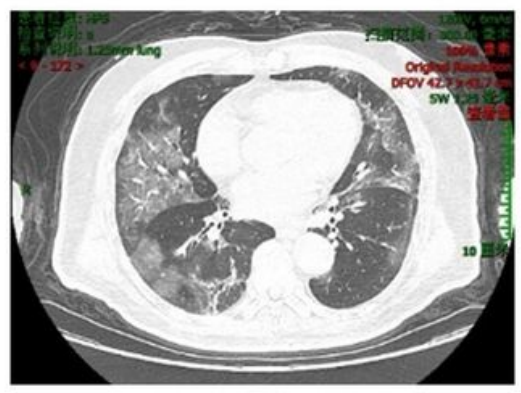

E

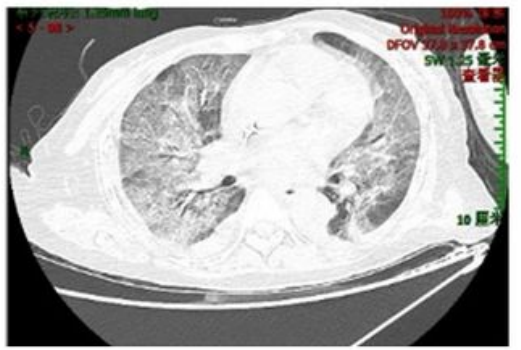

C

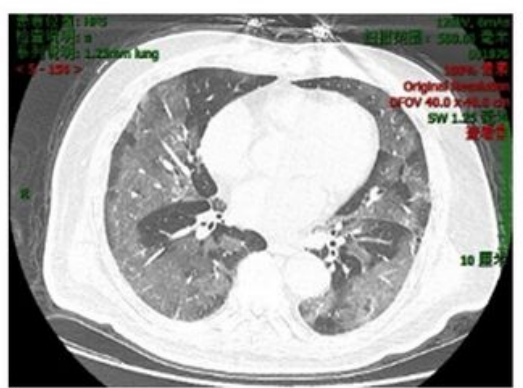

F

Figure 2

CT images from a female over 75-year-old $(A, B, C)$ and a male over 70-year-old (D, E, F) COVID-19 patients. (A) Day 6 after symptoms onset: multifocal patchy ground-glass opacities in bilateral subpleural areas. (B) Day 9 after symptoms onset: expansion of bilateral pulmonary lesions and denser pulmonary consolidation. (C) Day 15 after symptoms onset: extensive ground-glass opacities in both sides showing a white lung appearance with air bronchograms. The patient died 7 days after the final scan. (D) Day 7 after symptoms onset: multifocal patchy ground-glass opacities in bilateral areas. (E) Day 11 after symptoms onset: enlarged crescent ground-glass opacities in both subpleural areas. (F) Day 14 after symptoms onset: extensive ground-glass opacities with obvious air bronchograms in bilateral areas. The patient died of anteroseptal ST-segment elevation myocardial infarction 3 days after the final scan. 